\title{
Anabases
}

ANABASES Traditions et réceptions de l'Antiquité

$2 \mid 2005$

Varia

\section{Politiche, antiche e moderne}

\section{Paulo Butti de Lima}

\section{OpenEdition}

\section{Journals}

\section{Edizione digitale}

URL: http://journals.openedition.org/anabases/1709

DOI: 10.4000/anabases.1709

ISSN: 2256-9421

\section{Editore}

E.R.A.S.M.E.

\section{Edizione cartacea}

Data di pubblicazione: 1 ottobre 2005

Paginazione: 235-241

ISSN: 1774-4296

\section{Notizia bibliografica digitale}

Paulo Butti de Lima, «Politiche, antiche e moderne », Anabases [Online], 2 | 2005, Messo online il 01 juillet 2011, consultato il 20 octobre 2019. URL : http://journals.openedition.org/anabases/1709 DOI : 10.4000/anabases. 1709

Questo documento è stato generato automaticamente il 20 ottobre 2019.

(c) Anabases 


\title{
Politiche, antiche e moderne
}

\author{
Paulo Butti de Lima
}

1 Sulla scia degli «Ateliers» precedenti (Anabases I), ci faremo guidare in questa sezione dalla ricezione e dalla dossografia, dagli archivi, eventuali, e dalle novità. Sono modi per presentare quello che, con voluta ambiguità - rifacendoci alla «scrittura della storia » richiamata da Pascal Payen nel primo numero della rivista - chiameremo «le scritture della politica». La nostra attenzione si volgerà al modo in cui queste «scritture » si affermano a partire da un vocabolario e da un repertorio tematico e narrativo antico - un procedimento che è anche di selezione e codificazione di testi antichi, che parlano di "politica» ai loro lettori moderni e che, con movimento inverso, passano a parlare di ta politika ai loro primi lettori. Oggetto poco chiaro di discorso, dai confini imprecisi e continuamente attraversati : così già nella peculiarità « geografica » di alcune delle sue prime testimonianze - la politica al di fuori delle poleis - nonché nei generi letterari che lo "traducono». Rispetto alla ricezione delle idee accentueremo in questo processo la configurazione del genere - un genere «della politica » che trova un riscontro difficile e incerto negli autori che ne offrono la prima espressione.

2 1. L'Asia è « la véritable école, où nous devons aller puiser toutes nos connoissances en politique » : l'osservazione di Linguet ${ }^{1}$ potrebbe ben adattarsi a più di un momento della riflessione degli antichi Greci sulle " cose della città ». Più di una volta questa riflessione sembra costituirsi ancorandosi alle figure di alcuni grandi sovrani orientali. Proprio per il significato che hanno assunto nell'affermazione, consolidamento e, in certo modo, ricomposizione della dinastia achemenide, Ciro e Dario servono da modello e da fonte - di informazioni e immagini - per la considerazione della imposizione del dominio tra gli uomini e della distribuzione del potere nella comunità. Immagini e racconti che hanno origine fuori delle poleis e che si rendono, in questa appropriazione, " politiche ». Il buon governo di Ciro è, nell'antichità greca, fonte di svariate riflessioni, e Senofonte, con il suo romanzo "morale e politico", ne è solo la principale espressione, eccezionale comunque già nella forma letteraria. Ma la Ciropedia, come sappiamo, avrà un ruolo di eccezione anche come fonte per la riflessione successiva sull'azione e formazione dei governanti, ed è difficile misurarci con l'ampiezza della sua fortuna in età moderna, con il continuo riferimento ad essa come repertorio di esempi 
per la trattatistica politica ${ }^{2}$. Già la figura di Dario, le cui gesta ricorrono ugualmente in simili percorsi di appropriazione storico-politica, offre lo spazio - con la personale affermazione della monarchia, in seguito alla rottura delle successioni legittime e di fronte quindi ad un suggerito cambiamento nella forma di distribuzione del potere per la riflessione sulle politeiai, punto fermo per i Greci di ciò che è un logos sulle cose politiche. Questo Oriente direttamente trasposto nella polis è il contrario di quello spesso prefigurato negli studi sulla politica (o sul "politico»). Anche per questo, le figure di Ciro e di Dario che portano a riflettere sul mondo delle poleis appaiono, in parte, come invenzioni greche, e le loro parole e idee possono sembrare, per riprendere un'espressione significativa adoperata in un altro contesto, "fuori luogo ${ }^{3}$ ». Ma forse anche in questo caso, a ben vedere, le idee si trovano nel loro «luogo » : lo possiamo riscontrare evocando alcune riletture dell'affermazione del potere di Dario e del momento politico che lo precede.

3 2. Luciano Canfora ha richiamato di recente l'attenzione per il dibattito tra i congiurati persiani nel terzo libro delle Storie di Erodoto e la questione del governo democratico ${ }^{4}$. Se crediamo a Erodoto, di questo si sarebbe parlato in Persia, secondo una proposta presentata da Otane, più di un decennio prima delle riforme clisteniche (III, 80-83). L'affermazione erodotea provocava la reazione incredula dei suoi ascoltatori (VI, 43) e di buona parte dei suoi lettori successivi, che non hanno esitato a mettere l'accento sul carattere eminentemente greco di quella situazione, per noi « inaugurale » : il dibattito sulla politica e la distinzione, chiaramente argomentata, tra le forme di governo, con la singolare difesa della democrazia/isonomia da parte di un notabile persiano. La tesi della credibilità di Erodoto ha sempre trovato i suoi sostenitori e Canfora la riprende per le conseguenze politiche di quel paradosso : "Cosa sarà stata una "democrazia" in un regno di enormi proporzioni come l'impero persiano è difficile dire. Che questa tradizione avesse tuttavia un fondamento non è da escludere. Probabilmente il nobile persiano Otane, autore di tale proposta, intendeva propugnare un ritorno al costume di "uguaglianza" vigente nell'antica Perside [...] In epoca arcaica, nel Vicino Oriente, forme di rappresentanza nelle comunità locali possono aver costituito degli embrioni di procedure democratiche : riunioni della comunità, designazione di rappresentanti ${ }^{5}$. »

4 La fiducia nelle parole erodotee porta così a capovolgere le idee comuni e la percezione dei processi politici, sia che si tratti della « realtà » che resta dietro al dibattito, sia che si parli dell'intenzione dello storico nel riportarlo. Canfora rinvia, per le sue considerazioni, alle analisi di David Asheri su Erodoto, in particolare nell'edizione del terzo libro delle Storie di Erodoto presso la Fondazione Lorenzo Valla ${ }^{6}$. Sul tema Asheri era tornato anche in un testo su Lo Stato Persiano rimasto inedito e che solo ora vede la pubblicazione ${ }^{7}$. Asheri si pone a fianco di Erodoto, dal punto di vista della veridicità : non bisogna indulgere alle facili critiche degli studi sull'Erodoto «bugiardo ». Ma se è vero che il confronto con l'iscrizione di Behistun, come voleva lo studioso russo V. Struve ${ }^{8}$, mostra elementi di contatto con le dichiarazioni di Dario in Erodoto, può anche darsi che si tratti semplicemente di coincidenze tra affermazioni di carattere generico. In ogni caso, forme diverse di governo degli uomini, o almeno modi diversi di governo monarchico, potevano essere previste in ambiente orientale. " E' certamente doveroso porre in rilievo il fatto incontestabile che Erodoto conosceva perfettamente gli ideali monarchici di Dario [...]. Ciro, Cambise e Gaumata avevano impersonato, entro meno di trent'anni, tre forme diverse di monarchia imperiale: la regalità paternalistica, il dispotismo autoritario, la monarchia populista. » Pur reiterando la struttura completamente greca del racconto erodoteo, Asheri suggerisce che vi erano 
in Oriente le basi per quel "racconto ", e soprattutto accentua l'intenzione di Erodoto di contrapporsi alla pretesa greca o ateniese (Asheri parla della "posa altezzosa o nazionalista » dei Greci) della scoperta della democrazia.

5 3. "Capire » la democrazia greca attraverso le idee persiane, e non per contrasto, ma per trasposizione. Questa una delle singolari conseguenze della lettura del testo erodoteo, in particolare della lettura che condivide con l'autore la fiducia nell'autenticità del dibattito. La « democrazia » in Persia rappresenta il vero problema, per gli ascoltatori di Erodoto e per noi, suoi lettori. Tuttavia, anche l'idea di un dibattito sulle «forme di governo » rivendica, letterariamente, la stessa pretesa di grecità - ciò che coincide, in una certa misura, con l'attribuzione ai Greci della paternità della « riflessione politica ${ }^{9}$ ".

6 Il dibattito tra i congiurati persiani è, nella sua forma, un'eccezione al racconto erodoteo (come sarà poi il dialogo dei Meli in Tucidide). Si tratta di un dibattito assembleare, più che di un dialogo, anche se in un'assemblea invero ridotta - e dibattito dagli esiti prevedibili, procedimento di conferma della tradizione avita - lo dice Dario esplicitamente -, reiterazione della forma ideale del dominio, che nella sua perfezione (ma anche impossibile realizzazione) sarà riaffermata nella tradizione filosofica greca (come, ad esempio, nel Politico di Platone). I passaggi argomentativi successivi e necessari, nel dibattito erodoteo, e l'approdo scontato, rendono a questa inaugurazione della riflessione politica la forma del rituale. « Rituali » sembrano i procedimenti per la scelta di Dario, successivamente all'opzione per il governo monarchico. La prova del cavallo, che per primo, all'alba, nitrisce, è vinta da Dario con uno stratagemma, di cui Erodoto offre due versioni (III, 86-87). Si è potuto osservare che la figura del cavallo assume un particolare rilievo nelle antiche civiltà iraniche, e si è potuto aggiungere che vi erano racconti sulla scelta del re al sorgere del sole ${ }^{10}$. Con una analisi brillante, George Dumézil accosta al racconto erodoteo le pratiche vediche che riguardano la presenza del cavallo nei rituali regali. Nell'aívamedha il cavallo, lasciato libero per un anno, "qualifie son possesseur pour accomplir le sacrifice où il sera lui-même victime ». Nel vàjapeya il re che sacrifica è il vincitore di una corsa truccata di cavalli. Nota Dumézil che nel primo caso, prima di essere lasciato libero, il cavallo è oggetto di varie oblazioni tra cui alcune relative alla sua voce.

7 Dumézil non s'accontenta però di fornire elementi per la comprensione della scelta di Dario secondo forme rituali vediche. Anche il dibattito tra i congiurati viene ricondotto alla sequenza delle pratiche preparatorie per la scelta del re. « Ne serions-nous pas en présence d'un morceau de rituel d'intronisation simplement interprété par un Grec à l'usage des Grecs, et, du rang de rituel, promu (ou dégradé) à celui d'évènement historique "arrivé une fois" ?» La corsa dei carri, precedente l'intronizzazione del re nel vàjapeya, serve d'appoggio all'argomentazione di Dumézil. In uno dei carri è presente il re, nell'altro è presente, a scelta, un membro " dell'aristocrazia guerriera » oppure "un contadino libero ». Negli altri carri si trova soltanto il conduttore, a rappresentare non un gruppo sociale, ma il numero delle divisioni dell'anno. La corsa conduce necessariamente alla vittoria del re, come avviene nel dibattito precedente la scelta di Dario. La forma « rituale » del dibattito politico molto direbbe del suo rapporto con l'azione - le parole sembrano pronunciate indipendentemente dalla vera messa in gioco, l'imposizione del potere, o solo come preparazione alla sua ineluttabile conferma. Dumézil avrebbe potuto aggiungere che, secondo l'interpretazione erodotea (o della sua fonte), anche l'uccisione dei magi realizza il passaggio (inverso) tra fatto 
«storico » e forma rituale. Svincolare le parole sulla politica - sotto la forma del consiglio o deliberazione - dalle azioni degli uomini che comandano assume qui un particolare significato. Ma lo studioso si limita ad accennare, in conclusione, al legame che si è sviluppato, in Grecia, tra le teorie « dei regimi politici » e la teoria dei generi di vita nell'ambito delle scuole filosofiche.

4. Se si tratta di nascita della riflessione politica, allora non si può non rilevare che il dibattito erodoteo è anche il risultato del rovesciamento di una casta sacerdotale. Erodoto chiama i rivoltosi hoi epanastantes toisi magoisi, dopo avere ricordato la festa dei magophonia (III, 79). Dalle rovine delle leggi divine (e dalla soggezione dei suoi rappresentanti) nasce la discussione sulla politica o sulla filosofia politica. E' indispensabile " porre in dubbio l'autorità o liberarsi di essa, per la scoperta del diritto naturale ». Sono le forme di rottura con ciò che è diversamente giustificato, che non attraverso la ragione, a provocare la riflessione sull'imposizione del potere tra gli uomini. La condizione dialogica socratica e il dibattito erodoteo sono perciò ravvicinati nell'analisi di Leo Strauss. A rendere conto di un elemento non secondario della sua riflessione politica serve la struttura del racconto erodoteo, più che l'effettivo rovesciamento del potere in Persia. Il dibattito sulle politeiai non solo è situato in un mondo non greco, ma è una diretta conseguenza della distruzione del potere religioso. «Erodoto ha mostrato questa condizione di cose collocando fuori della Grecia il solo dibattito sui principi di politica riportato nelle sue Storie: espressamente egli ci dice che quella libera discussione è avvenuta nella Persia amante della verità dopo il massacro dei magi. » L'amore dei Persiani per la verità era stato affermato da Erodoto stesso (I, 136), tra i principi della paideia persiana. Ma sarebbe naturalmente improprio pensare alla monarchia di Dario come a un governo lontano dalla divinità, e in effetti Strauss aggiunge : «Con ciò non si nega che la nozione del diritto naturale, una volta emersa ed impostasi come cosa ovvia, possa facilmente accordarsi con la fede nell'esistenza di leggi divinamente rivelate. Solo affermiamo che il predominio di quella fede impedisce il sorgere della nozione di diritto naturale, o comunque rende la sua ricerca di importanza assolutamente secondaria ${ }^{11}$. $\gg$ Con il suo esempio, Leo Strauss rende al dibattito erodoteo un ruolo privilegiato all'origine di ciò che soltanto il dialogo socratico potrà fare apparire nella sua pienezza e che coincide con la formazione stessa di una riflessione politica.

9 5. Queste sono soltanto alcune delle riletture "politicamente » più significative che si sono fatte del dibattito erodoteo - ma sarebbe da rendere conto, all'indietro, della storia della lettura e ricezione di questo testo nelle riflessioni classiche sulle forme di governo. Possiamo qui solo accennare al fatto che la democrazia - che fa parte dei nostri interessi così come di quelli erodotei - non sempre ha costituito il problema principale per chi ha dedicato l'attenzione al dibattito. Nel corso del ' 700 , nell'ambito di studi orientali fortemente segnati dalle ricerche sullo zoroastrismo, è soprattutto la continuità monarchica a suscitare l'interesse degli uomini di lettere - a incominciare dalla figura di Ciro, infine trasformata da Voltaire in un esempio di tiranno (e un « fléau de la terre ${ }^{12} »$ ). Nella lettura del dibattito erodoteo viene quindi messa in evidenza la critica alla monarchia, una forma di governo, dice Charles Rollin nella sua Histoire Ancienne, della quale Otane " exagéra avec force les dangers et les inconvénients, tels, selon lui, sur-tout à cause du pouvoir absolu et sans bornes qui y est attaché, que le plus homme de bien ne peut pas tenir contre, et en est presque infailliblement renversé ${ }^{13}$ ». 
Inutile dire che gli altri interventi dei congiurati non ricevono da Rollin la stessa attenzione critica ${ }^{14}$.

Se Voltaire si riferiva, criticamente, al romanzo "morale e politico" di Senofonte, Chateaubriand si rivolgerà al quadro «morale e politico » della Persia, composto anche attraverso i racconti erodotei, per parlare degli accadimenti politici a lui contemporanei ${ }^{15}$. Ma i congiurati persiani non tramano, per Chateaubriand, una di quelle rivoluzioni di cui si occupa l'Essai. Appartengono a un mondo in crisi, in attesa dei venti greci rivoluzionari. Né si preparano ad una "controrivoluzione», come indicherà qualche studioso più recente ${ }^{16}$. Può comunque constatare Chateaubriand: "La théorie des gouvernements semble aussi avoir été familière aux sages de la Perse. " Il riferimento di Chateaubriand è in primo luogo alla figura di Zoroastro legislatore, e siamo nell'ambito degli interessi diffusi all'epoca ${ }^{17}$. Zoroastro legislatore non giustificava però la dimestichezza dei Persiani con le politeiai. Era quindi forzoso il riferimento al dibattito: "Hérodote introduit ailleurs les seigneurs persans, après l'assassinat du mage, délibérant sur le mode de gouvernement à adopter pour l'empire.» Come già avveniva nella Storia di Rollin, si guarda alla critica della monarchia da parte di Otane. Chateaubriand non esita a riferire le parole antitiranniche del Persiano : «Le tyran, dit-il, ta men gar, hybrei kekoremenos, erdei polla kai atasthala ta de phthono, tantôt gonflé de haine, tantôt d'orgueil, commet des actions horribles. » Nella revisione del 1826, Chateaubriand nulla aggiungerà, in questo passo, alla nota con il riferimento a Erodoto. Tuttavia, subito dopo farà la precisazione : « En lisant avec attention l'Essai, on découvre sous le rapport politique que mon dessein est de prouver, sans admettre et sans rejeter le gouvernement républicain en théorie, que la république ne pourrait s'établir en France, parce que les mœurs n'y sont plus assez innocentes [...]. Dans cet âge avancé du monde la liberté est plus aimable sous la forme monarchique que sous la forme républicaine, parce que le pouvoir exécutif placé dans une famille souveraine exclut les ambitions individuelles, toujours plus vives dans l'absence des mœurs. » La critica alla tirannide, da parte di Otane, si spegne così nel compiacimento per il governo monarchico nelle società avanzate, come quella persiana, corrotta e quindi non più innocente ( $«$ il est impossible de supposer que tant de lumières - presso i Persiani - pesassent dans un des bassins de la balance, sans un contrepoids égal de corruption »). Del gioco delle analogie, procedura storiografica abituale ${ }^{18}$, Chateaubriand spinge al massimo le ambiguità e le inversioni. Dai Persiani/ Tedeschi, in attesa della rivoluzione, alla conferma di Dario sul trono della Francia, il dibattito erodoteo ritrova la sua più singolare lettura tra gli esempi della saggezza persiana.

\section{NOTE}

1. Simon-Nicolas-Henri Linguet, Théorie des lois civiles, ou Principes fondamentaux de la société, Londres, 1767, vol. I, Discours préliminaire, p. 108 (citato da L. GUERCI, Libertà degli antichi e libertà dei moderni, Napoli, 1979, p. 149). 
2. Cf. C. GRELL, "Daniel, Hérodote, Xénophon: le grand Cyrus à l'âge classique, $\mathrm{XVI}^{\mathrm{e}}$-XVIII ${ }^{\mathrm{e}}$ siècles", Quaderni di Storia 20 (1984), p. 111-156 ; EAD., Le Dix-huitième siècle et l'Antiquité en France 1680-1789, Oxford, 1995, p. 342-351; 763-766, ecc.

3. L'espressione «idéias fora do lugar» è stata adoperata per lo studio delle idee liberali « trasposte » nella società schiavista brasiliana dell'Ottocento, con un riferimento particolare alle implicazioni di questa trasposizione per la composizione del romanzo brasiliano : in questo caso si trattava però della non aderenza di queste idee alla situazione sociale del paese e le conseguenze di questo divario nella scrittura letteraria: cf. in particolare, R. SCHWARTZ, Ao Vencedor as Batatas, São Paulo, 1977. Per l'espressione "as idéias estão no lugar », cf. M.S. DE CARVALHO FRANCo, “As idéias estão no lugar”, Cadernos de Debate I, São Paulo, 1976.

4. L. CANFoRA, La democrazia. Storia di un'ideologia, Roma-Bari, 2004 (traduzione francese presso Seuil, Paris, 2005). Si veda anche l'intervento dell'autore in Anabases 1 (2005), p. 258.

5. CANFORA, La democrazia, p. 31 e p. 32, con rinvio, per le assemblee iraniane, al lavoro di S.L. UTCENKO e I.M. DIAKONOV, Local Stratification of Ancient Society, Nauka Publ. House, Moscow, 1970.

6. D. ASHERI, Erodoto. Le Storie. Libro III, Milano, 1990. Cf. anche, dello stesso autore, "L'ideale monarchico di Dario : Erodoto III 80-82 e DNb Kent", AION (archeologia), 3 (1996), p. 99-106, dove il tema è sviluppato però con meno incisività dall'autore.

7. D. ASHERI, O Estado Persa, di prossima pubblicazione presso le edizioni Perspectiva, São Paulo, 2005.

8. V.V. STRUVE, "Gerodot i politiceskije tecenija v Persii epochi Darija I" ("Erodoto e le correnti politiche in Persia all'epoca di Dario I"), in Vestnik Drevnej Istorii 3 (1948), p. 12-35, commentato da ASHERI, in $O$ Estado Persa. Sempre in VDI 1 (1960), p. 20-34, B.B. Margules difende l'origine persiana del dibattito (apud A. Momigliano, Terzo contributo alla storia degli studi classici, Roma, 1966, p. 709, n.1). Cf. inoltre M.A. DANDAmaev, Persien unter den ersten Achämeniden (6. Jahrhundert v. Chr.), Wiesbaden, 1976 (dall'edizione russa del 1963), p. 163-166, e, dello stesso autore, A Political History of the Achaemenid Empire, Leiden - New York - Kobenhavn - Köln, 1989 (traduzione aggiornata dell'originale russo, Mosca, 1980), p. 105-106. A.R. BURN (Persia and the Greeks, London, 1984, 2nd ed., p. 95) ricorda che la monarchia assoluta « is not a primitively universal form of government » e che « the Persians were not many generations removed from tribal life... »

9. Già J. WelLs parlava a riguardo di «nascita della filosofia politica greca », pur accentuando la veridicità erodotea : cf. "The Persian Friends of Herodotus”, Journal of Hellenic Studies 27 (1907), p. 37-47 ; e W.W. HOW - J. WELLS, A Commentary on Herodotus, Oxford 1928, ad loc. Per l'importanza del dibattito in quanto riflessione politica cf. D. LANZA, Il tiranno e il suo pubblico, Torino, 1977, p. 228 (e in generale sul dibattito, p.225-232). Si veda inoltre F. LASSERRE, "Hérodote et Protagoras: le débat sur les constitutions”, Museum Helveticum 33 (1976), p. 65-84 ; F. HARTOG, Le

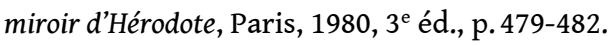

10. Cf. Ctesia, FGrHist., 688 F 13, 17. W. ALY, Volksmärchen, Sage und Novelle bei Herodot und seinen Zeitgenossen, Göttingen, 1969 (prima edizione 1921), p.97-107 (in part., p. 104 s.) ; D. LANZA, Il tiranno, p. 226 ; G. DUMÉZIL, "L'intronisation de Darius", in Orientalia. J. Duchesne-Guillemin emerito oblata, Leiden, 1984, p. 143-149 ; D. ASHERI, Erodoto. Le Storie. Libro III, p. 302 ; D. LENFANT, Ctésias de Cnide, ed. Belles-Lettres, Paris, 2004, p. LXXX-LXXXI.

11. L. STRAUSS, Diritto naturale e storia, trad. it. di N. Pierri, Genova, 1990, p. 94 s.

12. Così nell'Essai sur les Mours et l'Esprit des Nations (nell'edizione ginevrina del 1756 a p. 25) Ma Voltaire è spesso tornato sul tema: si veda in particolare la voce Cyrus nel Dictionnaire Philosophique.

13. Ch. Rollin, Historie Ancienne (ed. Letronne, Paris, Didot, 1821-1825), vol. 2, p. 242.

14. Altri, ancora prima di Rollin, si sono limitati a riprendere la narrazione del dibattito o a constatarne l'inverosimiglianza. Già Bayle, nel Dictionnaire, s.v. Darius, si occupava piuttosto di indicare gli errori commessi da Moreli (curiosamente Bayle non ha una voce su Ciro il Grande, ma 
solo su Ciro, il giovane). Commenta E. de Condillac a proposito del dibattito erodoteo (Introduction à l'Étude de l'Histoire Ancienne, nel Cours d'étude pour l'instruction du Duc de Parme, Parme, 1775, vol. 5, p. 159) : «Je soupçonne cet historien d'avoir saisi cette occasion pour dire ce qu'il pensoit sur chaque espèce de gouvernement. »

15. Essai historique, politique et moral sur les révolutions anciennes et modernes, considérées dans leurs rapports avec la Révolution française, prima ed. Londres, 1797 ; seconda ed., con l'aggiunta di note, Paris, 1826 (per quanto qui riportato, cf. $1^{\text {re }}$ Partie, chap. LIX). Sull'Essai e sul rapporto tra le due edizioni resta sempre importante P. VIDAL-NAQUET - N. LORAUX, La formation de l'Athènes bourgeoise (1979), in P. VIDAL-NAQUET, La démocratie grecque vue d'ailleurs, Paris, 1990, p. 183-188, Su Chateaubriand storico è ora da vedere F. HARTOG, Régimes d'historicité, Paris, 2003, p. 77-107.

16. Così BURN, Persia, p. 94-95.

17. Nei Mémoires de l'Académie des Inscriptions et Belles-Lettres del 1774 (t. 37, p. 710 ss.) viene pubblicata la relazione di Anquetil du Perron, letta nel 1769, intitolata: Recherches sur le temps auquel a vécu Zoroastre, législateur des Perses, et auteur des livres Zends.

18. Cf. Voltaire, Dictionnaire philosophique, s.v. Xénophon : «Les Grecs étaient donc précisément ce que sont aujourd'hui les Helvétiens, qui louent leur service et leur courage aux princes leurs voisins. »

\section{AUTORE}

\section{PAULO BUTTI DE LIMA}

Università degli studi di Bari

butti@lettere.uniba.it 